\title{
La trimérisation spontanée des membres de la famille des récepteurs du TNF
}

Décrite pour la première fois en 1975, la cytokine TNF (tumor necrosis factor $\alpha$ ) joue un rôle essentiel dans la réponse inflammatoire et la réponse immunitaire. Cette cytokine n'a aucune activité enzymatique. Elle exerce ses effets sur les cellules par l'intermédiaire de deux récepteurs membranaires, TNFR1 (CD120a, $55 \mathrm{kDa}$ ) et TNFR2 (CD120b, $75 \mathrm{kDa})$. Au cours de ces dernières années, de nombreux récepteurs homologues ont été identifiés. Ils ont en commun, au niveau de leur domaine extracellulaire, des motifs riches en cystéines (CRD ou cysteine-rich domains) qui participent à leur interaction avec leur ligand respectif. Leur domaine intracellulaire est dépourvu de toute activité enzymatique, mais l'interaction du récepteur avec son ligand spécifique provoque le recrutement de protéines cytosoliques qualifiées de protéines adaptatrices, par l'intermédiaire de domaines d'interaction protéine-protéine. La nature de la protéine recrutée détermine la réponse cellulaire à l'interaction ligand-récepteur.

Certains récepteurs de la famille des récepteurs du TNF sont caractérisés par la présence, dans leur domaine cytoplasmique, d'une séquence d'environ 80 acides aminés appelée " domaine de mort». Ce motif peut interagir avec un motif homologue des protéines adaptatrices appelées TRADD (TNF receptor-associated death domain protein) ou FADD (Fas-associated death domain protein). Les récepteurs comportant un domaine de mort sont notamment TNFR1, Fas (CD95/APO-1), et les récepteurs de TRAIL (TNF-related apoptosis inducing ligand), DR4 et DR5 (death receptor). Un second groupe de récepteurs du TNF est constitué de protéines homologues à TNFR2, et caractérisées par un motif moins bien défini de leur domaine cytoplasmique qui se lie à des molécules adaptatrices de la famille des TRAF (TNF-receptor-associated factor) [1].
Les ligands de la famille du TNF existent sous forme membranaire ou soluble. Leur forme active est constituée de l'association de trois molécules identiques. Cet homotrimère doit interagir avec un récepteur homotrimérique pour déclencher une cascade de signalisation dans la cellule [1]. On pensait jusqu'alors que le ligand homotrimérique provoquait l'agrégation des monomères de récepteur. Concernant Fas, cette oligomérisation, en rapprochant trois domaines de mort dans le cytosol, était supposée permettre le recrutement de la molécule adaptatrice FADD qui, elle-même, recrutait la procaspase-8 dont l'activation déclenche la cascade d'évènements conduisant à l'apoptose (figure 1A). Deux publications récentes suggèrent que l'agrégation de Fas et des autres membres de la famille récepteurs du TNF existe avant toute interaction avec leur ligand [2, 3], et qu'elle serait même un pré-requis à l'interaction ligand-récepteur (figure 1B).

L'oligomérisation de Fas fait intervenir un domaine amino-terminal extracellulaire appelé PLAD (pre-ligand-binding assembly domain) [2]. Ce domaine est, bien entendu, distinct des domaines d'association des protéines adaptatrices qui sont cytoplasmiques, mais aussi des domaines d'interaction avec le ligand spécifique. Un PLAD a été identifié au niveau de la partie extracellulaire de plusieurs autres membres de la famille des récepteurs du TNF, TNFR1, TNFR2, CD40, et DR4 $[2,3,4]$, ce qui suggère qu'il s'agit d'une caractéristique commune à l'ensemble des membres de cette famille. En outre, chaque PLAD est spécifique de son récepteur, permettant ainsi d'éviter toute oligomérisation entre des récepteurs non homologues [2]. L'agrégation de récepteurs membranaires, avant toute interaction avec le ligand, a déjà été décrite pour d'autres familles de récepteurs membranaires, comme ceux de l'IL-1 et de l'IL-2 (formés de l'assemblage de polypeptides distincts) et celui du récepteur de l'érythropoiétine sous forme d'homodimère $\left(\mathrm{m} / \mathrm{s} 1999, n^{\circ} 8 / 9, p\right.$. 1049). Pour ce dernier, l'association des deux monomères fait intervenir le même domaine que celui qui est impliqué dans la liaison du ligand [5].

Ces résultats ont aussi permis de mieux comprendre la pathogénie du syndrome lymphoprolifératif autoimmun, également connu sous le nom de maladie de Canale-Smith [3]. Cette affection héréditaire rare se traduit dès la petite enfance par une accumulation de lymphocytes polyclonaux. Les manifestations cliniques associent des adénopathies, une hépatosplénomégalie et des manifestations auto-immunes. Ce syndrome est dû à un défaut d'apoptose lymphocytaire provoquant l'accumulation de lymphocytes $\mathrm{T}$ double négatifs. Une des formes de ce syndrome (le type Ia) est le plus souvent la conséquence d'une mutation hétérozygote du gène APT1 codant pour le récepteur Fas [6], et la plupart des mutations identifiées sont des mutations ponctuelles affectant le domaine de mort. Des expériences de transfection in vitro ont montré que les protéines Fas dont le domaine de mort est muté se comportent de manière dominante négative : la protéine mutée inhibe en effet la transmission du signal de mort par la protéine normale. Ceci est dû au fait que les motifs PLAD assurent l'assemblage de molécules Fas sauvages avec des molécules Fas mutées au sein des trimères [3]. Même si aucune mutation du domaine PLAD n'a été jusqu'à présent décrite, on peut penser que de telles mutations pourraient aussi être responsables de ce syndrome lymphoprolifératif puisque l'agrégation de Fas semble indispensable à son interaction avec FasL. Chez les sujets normaux (gène APT1 non muté), la trimérisation du récepteur Fas peut impliquer des molécules Fas tron- 


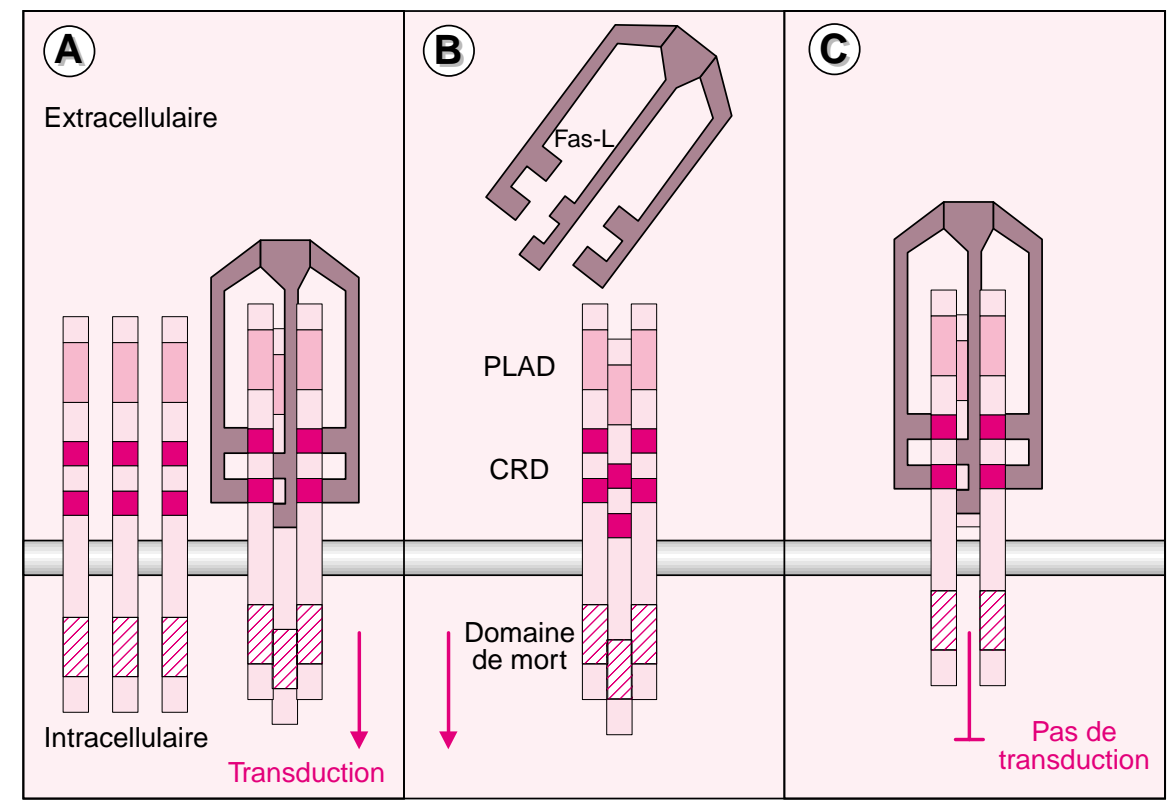

Figure 1. La trimérisation de Fas. La formation de trimères implique un nouveau domaine appelé PLAD localisé dans sa partie extracellulaire et distinct des domaines riches en cystéine (CRD) impliqués dans l'interaction avec le ligand. Dans la partie cytoplasmique de Fas, se trouve le domaine de mort à partir duquel sont activées les voies de signalisation intracellulaire. II existe maintenant deux modèles de trimérisation des récepteurs de la famille TNFR. A. Dans le modèle le plus ancien, l'interaction ligand-récepteur provoque la trimérisation du récepteur et la transduction du signal. $B$. Les travaux récents $[2,3]$ montrent qu'au moins une partie de ces récepteurs existent, avant toute interaction, avec le ligand sous forme de trimères dans les cellules. $\boldsymbol{C}$. Quel que soit le modèle, I'implication d'une isoforme tronquée de Fas dans le trimère inhibe la signalisation déclenchée par l'interaction de Fas avec son ligand.

quées. Ce variant soluble de Fas, issu d'un épissage alternatif de l'ARNm APT sauvage, est dépourvu de domaine de mort et interviendrait dans la régulation de la voie de signalisation de Fas [7].

Même si Fas existe sous forme de trimère, aucun signal de mort ne semble transduit tant que le ligand n'interagit pas avec le récepteur. En effet, en l'absence d'interaction avec FasL, il semble que les homotrimères Fas ne recrutent que partiellement la molécule FADD et ne recrutent pas $\mathrm{du}$ tout la procaspase- 8 puisque aucun clivage de la poly(ADPribose) polymérase, une cible des caspases effectrices, n'est identifiée [3]. Les événements déclenchés par la liaison de FasL aux trimères préformés de Fas restent hypothétiques. L'interaction ligand-récepteur pourrait moduler celle du récepteur avec des molécules inhibitrices comme
TNFR1). Dans l'éditorial de Science [8] associé à ces deux publications récentes [2, 3], Pierre Golstein propose deux autres hypothèses : l'interaction de FasL avec les trimères de Fas pourrait induire soit un changement de conformation de ces trimères, soit l'association de plusieurs trimères pour former des oligomères de plus grande taille. Cette dernière hypothèse pourrait expliquer le fait que, dans certaines lignées cellulaires tumorales, les agents cytotoxiques qui augmentent l'expression de Fas provoquent, même en l'absence de FasL, le recrutement de FADD et de la procaspase-8 et activent la cascade des caspases ([9] et $m / s ~ 1999, n^{\circ} 6-7$, p. 897). L'augmentation de la densité de Fas à la surface des cellules pourrait favoriser l'assemblage de multiples trimères de Fas, comme le font les rayons ultraviolets [10], déclenchant le recrutement de FADD et de la procaspase-8.
Il apparaît donc que ces domaines PLAD récemment identifiés sur les membres de la famille des récepteurs du TNF sont indispensables à l'homotrimérisation des récepteurs, elle même nécessaire à la transduction $\mathrm{du}$ signal, notamment en réponse à l'interaction ligand-récepteur. Ces domaines sont indépendants des domaines de mort et d'interaction avec le ligand. Ils constituent donc une nouvelle cible pour tenter de moduler la signalisation transduite par les cytokines interagissant avec ces récepteurs, ce qui pourrait avoir des applications en thérapeutique anti-inflammatoire et immuno-modulatrice.

1. Ledgerwood EC, Pober JS, Bradley JR. Recent advances in the molecular basis of TNF signal transduction. Lab Invest $1999 ; 79$ : 1041-50.

2. Chan FK-M, Chun HJ, Zheng L, Siegel RM, Bui KL, Lenardo MJ. A domain in TNF receptors that mediates ligand-independent receptor assembly and signaling. Science $2000 ; 288: 2351-4$.

3. Siegel RM, Frederiksen JK, Zacharias DA, et al. Fas preassociation required for apoptosis signaling and dominant inhibition by pathogenic mutations. Science $2000 ; 288: 2354-7$.

4. Papoff G, Hausler P, Eramo A, et al. Identification and characterization of a ligand-independent oligomerization domain in the extracellular region of the CD95 death receptor. J Biol Chem $1999 ; 274: 38241-50$.

5. Philo JS, Aoki KH, Arakawa T, Narhi LO, Wen J. Dimerization of the extracellular domain of the erythropoietin (EPO) receptor by EPO: one highaffinity and one low-affinity interaction. Biochemistry 1996 ; 35 : 1681-91

6. Rieux-Laucat F, Le Deist F, Hivroz C, et al. Mutations in Fas-associated with human lymphoproliferative syndrome and autoimmunity. Science $1995 ; 268: 1347-9$.

7. Cascino I, Papoff G, De Maria R, Testi R, Ruberti G. Fas/Apo-1 (CD95) receptor lacking the intracytoplasmic signaling domain protects tumor cells from Fas-mediated apoptosis. J Immunol 1996 ; 156 : 4622-30.

8. Golstein P. Fas binds preassembled Fas. Science $2000 ; 288: 2328-9$.

9. Micheau O, Solary E, Hammann A, DimancheBoitrel MT. Fas ligand-independent, FADDmediated activation of the Fas death pathway by anticancer drugs. J Biol Chem $1999 ; 274$ : 7987-92. 10. Aragane Y, Kulms D, Metze D, et al. Ultraviolet light induces apoptosis via direct activation of CD95 (Fas/APO-1) independently of its ligand CD95L. J Cell Biol 1998 ; 140 : 171-82.

\section{Éric Solary}

Marie-Thérèse Dimanche Boitrel Ali Bettaieb

Inserm U. 517, Facultés de médecine et pharmacie, 7, boulevard Jeanne-d'Arc, 21000 Dijon, France. 